\title{
Presencia de anticuerpos contra neurorreceptores cardiacos de acetilcolina muscarínicos tipo II en pacientes con enfermedad de Chagas e implantación de marcapasos
}

\author{
Nubia Catalina Tovar ${ }^{1}$, María Clara Echeverry ${ }^{1}$, Guillermo Mora ${ }^{2}$ \\ 1 Laboratorio de Parasitología, Departamento de Salud Pública, Facultad de Medicina, Universidad \\ Nacional de Colombia, Bogotá, D.C., Colombia \\ 2 Departamento de Medicina, Facultad de Medicina, Universidad Nacional de Colombia, Bogotá, D.C., \\ Colombia
}

Introducción. En diferentes tipos de afecciones cardiacas, incluida la enfermedad de Chagas, se ha descrito la presencia de anticuerpos que reconocen neurorreceptores cardiacos. La respuesta más frecuente es contra receptores de acetilcolina del tipo muscarínico subtipo II (anti-m2MAChR), que reconocen este receptor.

Objetivo. El objetivo del presente estudio fue establecer la frecuencia de anticuerpos antim2MAChR en un grupo de pacientes cardiópatas con implantación de marcapasos y enfermedad de Chagas.

Materiales y métodos. Cincuenta y dos pacientes con enfermedad de Chagas e implantación de marcapasos cardiaco fueron pareados por diagnóstico de implantación con 52 individuos con marcapaso y sin enfermedad de Chagas. La presencia de anticuerpos anti-m2MAChR fue estimada mediante ELISA e inmunoblot.

Resultados. El $32,7 \%$ de los casos presentaban anticuerpos contra el segundo dominio extracelular del receptor versus $3,8 \%$ de los controles $(p<0,001)$. El $51,9 \%$ de los casos presentaron anticuerpos contra el tercer dominio intracelular del receptor versus $19,2 \%$ de los controles $(p<0,001)$. No se encontraron diferencias clínicas entre los pacientes que presentan y los que no presentan anticuerpos anti-m2MAChR.

Conclusión. El odds ratio (OR) del presente estudio muestra una mayor probabilidad de presentar anticuerpos anti-m2MAChR en pacientes con implantación de marcapaso e infección por Trypanosoma cruzi, que en aquéllos con implantación que no presentan infección por el parásito. La presencia de anticuerpos anti-m2MAChR no está relacionada con ningún tipo de manifestación clínica de las evaluadas en este estudio.

Palabras clave: enfermedad de Chagas, Trypanosoma cruzi, receptores muscarínicos, marcapaso artificial, anticuerpos, corazón.

Presence of antibodies to cardiac neuroreceptors in patients with Chagas disease

Introduction. The presence of antibodies against cardiac neuroreceptors has been established in several kinds of heart diseases as well as in Chagas disease. The antibody type most frequently identified is that which recognizes the muscarinic acetyl choline receptor type II (antim2MAChR).

Objective. The frequency of the anti-m2MAChR was determined in a group of Colombian patients with permanent pacemaker implantation and Chagas disease.

Materials and methods. Fifty-two patients with Chagas disease and permanent heart pacemaker implantation were matched by implantation diagnosis with 52 individuals that required pacemaker, but without Chagas disease. The presence of antibodies that recognized the m2MACh was assessed in the two groups by ELISA and Western blot by using two peptide sequences of the (m2MAChR), the second extracellular domain (2e) and the third intracellular domain (3i).

Results. Serological response frequency against 2 e-m2MAChR in Chagas patients was $32.7 \%$ compared with $3.8 \%(p<0.01)$ in the controls; response against 3i-m2MAChR was $51.9 \%$ compared with $19.2 \%(p<0.01)$ for the controls. No clinical differences were observed between 
individuals that presented anti-m2MAChR and those who did not.

Conclusion. The frequency of anti-m2MAChR was higher in patients with Chagas disease for two of the receptor domains. Furthermore, patients with pacemaker therapy are more likely to have anti-m2MAChR and infection by Trypanosoma cruzi. The anti-m2MAChR response is not associated with any discernable clinical manifestation in this group of patients.

Key words: Chagas disease, Trypanosoma cruzi; receptors, muscarinic; pacemaker, artificial; antibodies, heart.

La regulación de la frecuencia y el inotropismo cardiaco la ejerce el sistema nervioso autónomo. Mediante este sistema, el gasto cardíaco responde a las condiciones fisiológicas variables del organismo. Las terminales del sistema nervioso autónomo del miocardio tienen neurorreceptores adrenérgicos ( $a$ y b adrenérgicos) y colinérgicos (muscarínicos) (1) que se encuentran presentes en cardiomiocitos y células ganglionares en proporciones diversas $(2,3)$. Estos son del tipo de receptores acoplados a proteínas G (G-protein coupled receptors, GPCR), en los cuales el sitio de interacción con el agonista se encuentra en el segundo dominio extracelular y, el acople a la proteína G, en el tercer dominio intracelular (4).

En pacientes con enfermedad de Chagas con cardiopatía crónica y en fase indeterminada, se ha descrito la presencia de anticuerpos circulantes que reconocen los neurorreceptores $\beta$-adrenérgicos y colinérgicos muscarínicos cardíacos del tipo II (anti-m2MAChR) que tienen actividad funcional en modelos in vitro e in vivo (5-11).

La reactividad de los anticuerpos anti-m2MAChR ha sido descrita contra el tercer dominio intracelular del receptor (12), pero la mayoría de estudios se han centrado en la reactividad dada por el reconocimiento de una secuencia de 25 aminoácidos correspondiente al segundo aro extracelular del m2-MAChR $(6,8,11,13)$ (figura 1). En ensayos in vitro, se ha evaluado la capacidad funcional de este reconocimiento

\section{Correspondencia:}

María Clara Echeverry, División de Parasitología, Facultad de Medicina, Universidad Nacional de Colombia, edificio 471, oficina 302, Ciudad Universitaria, Bogotá, D.C., Colombia. Teléfono: (571) 316 5000, extensión 15032 y 15033: fax: (571) 3165000 , extensión 15033

mcecheverryg@unal.edu.co

Recibido: 20/06/08; aceptado:13/03/09 y se ha demostrado que el acople del receptor con los anticuerpos produce una respuesta fisiológica similar a la del ligando natural, dada por la disminución en la fuerza de contracción del tejido cardiaco (14), la variación en los niveles intracelulares de AMPc y GMPc $(5,8-10)$, la supresión de corrientes de calcio $(15,16)$ y la pérdida de la sensibilización del receptor (11).

Esto ha permitido postular una posible asociación entre el desarrollo de la cardiopatía y la presencia de anticuerpos que interactúan con los neurorreceptores. No obstante, la evidencia al respecto es contradictoria. Mientras algunos estudios concluyen que la presencia de anticuerpos anti-m2MAChR en pacientes con cardiopatía chagásica crónica está asociada a manifestaciones clínicas de bradicardia (5), disfunción del nervio vago (17), fibrilación auricular (13), enfermedad del nódulo (18) y arritmias ventriculares (19), otros demuestran que no existe disfunción cardíaca en los pacientes que presentan altos títulos de estos anticuerpos (20).

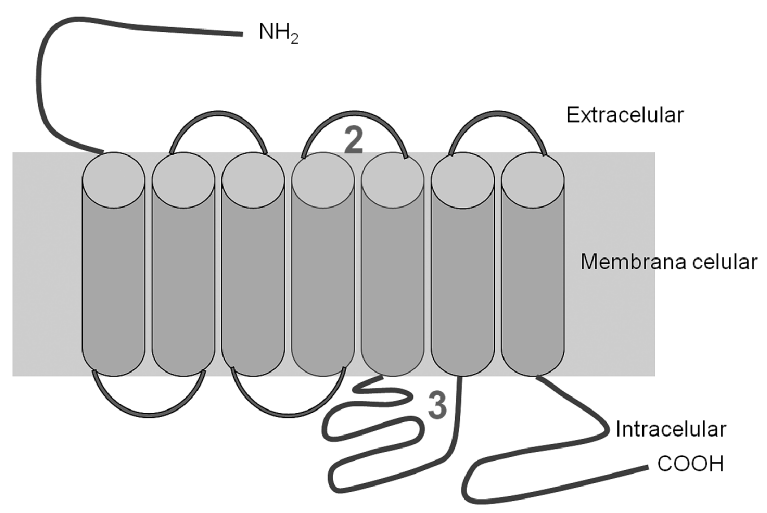

Figura 1. Diagrama del receptor muscarínico de acetilcolina tipo II. El número 2 señala el segundo aro extracelular del receptor; el número 3 señala el tercer aro intracelular de receptor. 
No obstante, la significancia de estos anticuerpos durante la infección por Trypanosoma cruzi, su grado de asociación con la enfermedad de Chagas y su utilidad pronóstica, permanecen sin ser aclarados.

El objetivo del presente estudio fue determinar la frecuencia de anticuerpos contra el tercer domino intracelular (tdi-m2MAChR) y el segundo dominio extracelular del receptor (sde-m2MAChR), en una población de pacientes con cardiopatía chagásica crónica e infección por $T$. cruzi, en comparación con pacientes con cardiopatía de otro origen.

\section{Materiales y métodos}

\section{Pacientes}

El presente estudio corresponde a un estudio descriptivo, retrospectivo, con diseño de casos (52 pacientes serológicamente positivos para $T$. cruzi) y 52 controles (con serología negativa), apareados por el diagnóstico clínico que se hizo para adelantar la implantación del marcapasos.

Los pacientes fueron seleccionados de un estudio previo en el que se les practicaron pruebas serológicas para T. cruzi por las técnicas de IFI y ELISA, y se clasificaron clínicamente (21). Para la realización del estudio, se contó con el aval del Comité de Ética de la Facultad de Medicina de la Universidad Nacional de Colombia y la firma del consentimiento informado de cada uno de los pacientes, de acuerdo con lo reglamentado por la Resolución 008430 de 1993 del Ministerio de Salud.

\section{Materiales}

Péptidos: se sintetizó un péptido correspondiente a la secuencia de aminoácidos del segundo aro extracelular del receptor de 168-VRTVEDGECYI QFFSNAAVTFGTAI-192, cuya identidad fue confirmada por espectrometría de masas ESI en la Emory University, PSN: 4653. Una proteína recombinante conformada por una proteína de fusión GST y un dominio correspondiente al tercer dominio intracelular del m2MAChR: 225VEDGECYIQFFSNAAVTFGTAIAAFYLPVIIMTV LYWHISRASKSRIKKDKKEPWANQDPVSPSLV QGRIVKPNNNNMPSSDDGLEHNKIQNGKAPR
DPVTENCVQGEEKESSNDSTSVSAVASNMRD DEI-356 (Acceso P08172)referencia AB5166 de la casa comercial Chemicon.

Los anticuerpos primarios correspondieron a un anticuerpo monoclonal contra el m2MAChR (22) y un anticuerpo policlonal contra el tdim2MAChR, referencia AB5166 marca Chemicon. Los anticuerpos secundarios se obtuvieron de la casa comercial ZIMED ref. 62-6520 y 627120. Kit Vector VIP (Vector Laboratories Ref: SK-4600). Los materiales de electroforesis y Western blot fueron suministrados por Biorad $y$, los demás reactivos, por SIGMA.

\section{Determinación de anticuerpos contra el tercer dominio intracelular m2MAChR (tdi-m2MAChR) por Western blot}

La proteína recombinante, referencia AB5166, descrita previamente y que contenía el tdim2MAChR, fue sometida a SDS-PAGE en un gel de poliacrilamida al 10\% usando $100 \mathrm{ng}$ por carril. Luego de la transferencia a papel de nitrocelulosa, se llevó a cabo el inmunoblot. La membrana fue bloqueada con una solución de TTBS (Tris- $\mathrm{HCl} 100 \mathrm{mM} \mathrm{pH} \mathrm{7,5,} \mathrm{0,9 \%} \mathrm{(w/v)}$ $\mathrm{NaCl}, 0,1 \%$ tween 20) con suplemento de leche descremada al $5 \%$ e incubada por una hora a temperatura ambiente.

Como control positivo se utilizó un anticuerpo policlonal contra el m2MAChR diluido 1:200 en TTBS con suplemento; los sueros de los pacientes fueron diluidos 1:20 en la misma solución.

El anticuerpo primario se incubó por 18 horas a $4^{\circ} \mathrm{C}$ con agitación constante, seguido de tres lavados con TTBS, de 10 minutos cada uno. El anticuerpo secundario anti-humano marcado con peroxidasa de rábano, fue diluido 1:3000 en TTBS con suplemento e incubado por una hora a temperatura ambiente con agitación constante; luego de lavada la membrana, la reacción se reveló colorimétricamente usando como sustrato el estuche Vector VIP para peroxidasa.

Como controles negativos se utilizaron sueros de cinco donantes sanos y con serología negativa para T. cruzi. Se consideró como resultado positivo la aparición de una banda a la misma altura del control positivo (50 kDa) (figura 1 y 2). 


\section{Determinación de anticuerpos contra el segundo dominio extracelular m2MAChR (sdi-m2MAChR) por ELISA}

Se dispusieron $50 \mu \mathrm{l}$ de una solución del péptido correspondiente al segundo aro extracelular del m2MAChR, a una concentración de $50 \mu \mathrm{g} / \mathrm{ml}$ en solución tampón de $100 \mathrm{mM}$ de $\mathrm{Na}_{2} \mathrm{CO}_{3} \mathrm{pH}$ 11,3 , en cada pozo en una placa de 96 pozos. Se incubó 18 horas a $4^{\circ} \mathrm{C}$. Luego de una hora de bloqueo (PBS: $10 \mathrm{mM}$ de fosfatos, $140 \mathrm{mM}$ de $\mathrm{NaCl} \mathrm{pH} \mathrm{7,3;} \mathrm{con} \mathrm{suplemento} \mathrm{de} \mathrm{tween} \mathrm{al} \mathrm{0,2 \% ,}$ $1 \%$ de BSA), las placas se incubaron 18 horas con los sueros de los pacientes diluidos 1:60 en solución de bloqueo a $4^{\circ} \mathrm{C}$.

Después de los lavados, se incubó el anticuerpo secundario anti-humano marcado con peroxidasa de rábano diluido 1:3000 en solución de bloqueo e incubado una hora a temperatura ambiente. La reacción colorimétrica se generó utilizando como sustrato TMB y la absorbancia se midió mediante un espectrofotómetro con

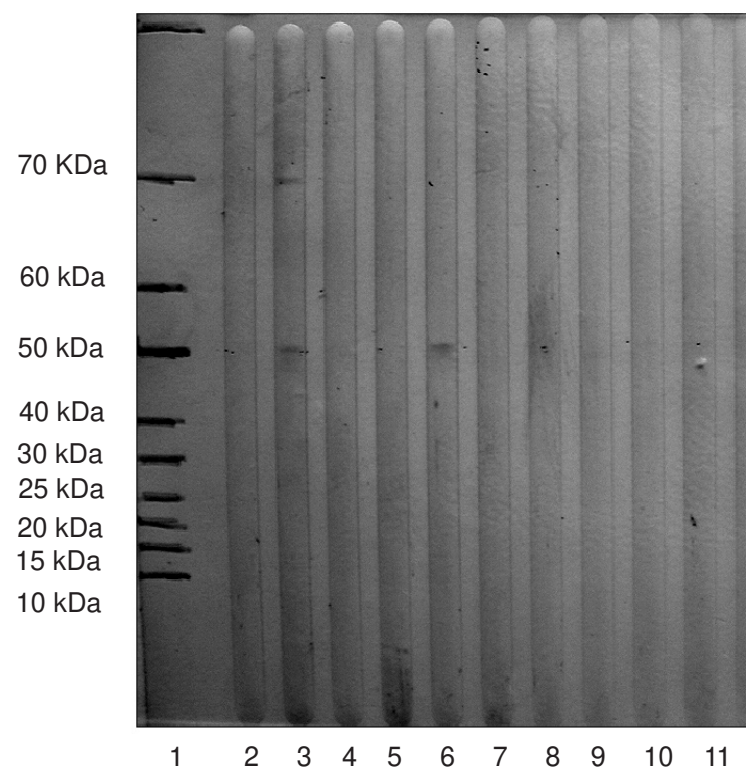

Figura 2. Determinación de anticuerpos contra el tercer dominio intracelular m2MAChR (tdi-m2MAChR) por inmunoblot; se presenta una fotografía de una membrana cuya reacción se ha revelado por el método colorimétrico, con reacción considerada como positiva contra el tdi-m2MAChR para el suero probado en el carril 6. En los carriles se encuentran los resultados de: 1, marcador de peso molecular; 2, control negativo; 3 , control positivo, y 4-11, sueros de pacientes. longitud de onda de $450 \mathrm{~nm}$ (9). Como controles negativos se utilizaron los mismos del inmunoblot. Se consideró como positiva la absorbancia 2,5 veces mayor que el promedio de la absorbancia de los controles negativos (13).

\section{Análisis estadístico}

La evaluación estadística se hizo por la prueba de ji al cuadrado con un nivel de confianza del $95 \%$, analizando la distribución de anti-m2MAChR entre casos y controles. Se compararon las variables demográficas y clínicas entre casos y controles, y entre individuos con anticuerpos anti-m2MAChR para alguno de los dominios del receptor e individuos que no presentaron respuesta. La información se analizó mediante las herramientas dispuestas en Epi-Info, versión 3.3.2.

\section{Resultados}

El grupo de estudio estuvo conformado por $61,5 \%$ mujeres, con un promedio de edad de 60 y 59 años para los casos y controles, respectivamente, y un $38,5 \%$ de hombres, con un promedio de edad de 56 y 58 años para casos y controles, respectivamente. La distribución geográfica de la población en estudio fue heterogénea; en el grupo de casos, la mayoría provenía del departamento de Boyacá y, en el grupo de controles, la mayoría pertenecía al departamento de Cundinamarca (no se presentan los datos).

Se evidenció que el mayor porcentaje de pacientes presentaron diversos tipos de bloqueos aurículo-ventriculares como motivo para la implantación del marcapasos. En el grupo de los casos, el síntoma más frecuente antes de la implantación del marcapaso fue la disnea, mientras que, en el de los controles, fue el síncope. La enfermedad asociada más frecuentemente en el grupo de casos fue la hipertensión arterial sistémica y, en grupo de controles, fue la dislipidemia (cuadro 1). No hubo diferencias estadísticamente significativas en la distribución de estas variables comparando casos y controles, ni comparando los pacientes que presentaron anticuerpos m2MAChR con los que no los presentaron. 
La evaluación de la presencia de anticuerpos contra los dominios funcionales del m2MAChR (figura 1) se adelantó por el método de inmunoblot, para identificar reacciones contra el tercer dominio intracelular; en el carril 6 de la figura 2 se aprecia un ejemplo de lo que se consideró como una reacción positiva en esta prueba. El método ELISA se utilizó para identificar reacciones contra el segundo dominio extracelular. En la figura 3 se presentan los valores de las densidades ópticas obtenidas por ELISA. Debido a que se determinaron como positivos aquellos sueros que presentaron un valor de absorbancia 2,5 veces mayor que el promedio de los controles negativos de cada ensayo, en la figura se aprecia cómo algunos de los puntos que se consideran como negativos tienen densidades ópticas mayores que las de la mayoría de los positivos.

En el grupo de los casos, 51,9\% (27 pacientes) presentó una reacción positiva contra el tercer dominio del receptor versus $19,2 \%$ (10 pacientes) de los controles (cuadro 2). En los casos, 32,7\% (17) de los pacientes presentó anticuerpos contra el segundo dominio extracelular del receptor Vs. 3,8\% (2) de los controles. El 15,4\% (8) de los casos presentaron anticuerpos para los dos dominios evaluados versus $1,9 \%$ (1) de los controles (cuadro 2). Se encontró una mayor probabilidad de presentar simultáneamente anticuerpos anti-m2MAChR contra los dos dominios en los casos que en los controles, como se muestra en el cuadro 2.

Debido a que el grupo de pacientes correspondía a quienes se les había implantado marcapasos por causas diversas, para evitar sesgos en el análisis por el diagnóstico de implantación del marcapaso, el grupo fue pareado por este factor. La distribución de esta variable evidencia que $71,1 \%$ de los pacientes tenía bloqueo aurículoventricular como causa de la implantación

Cuadro 1. Variables demográficas y clínicas de la población de estudio. Se describen las frecuencias simples para cada una de las variables evaluadas. Esta distribución de frecuencias no presenta significancia estadística en casos Vs. controles y, tampoco, en presencia Vs. ausencia de anticuerpos anti-m2MAChR.

\begin{tabular}{|c|c|c|c|c|c|c|}
\hline \multirow[t]{2}{*}{$\begin{array}{c}\text { Variables demográficas } \\
\text { y clínicas }\end{array}$} & & \multicolumn{2}{|c|}{$\begin{array}{l}\text { Enfermedad } \\
\text { de Chagas } \\
(n=52)\end{array}$} & \multicolumn{2}{|c|}{$\begin{array}{c}\text { Controles } \\
(n=52)\end{array}$} & \multirow[t]{2}{*}{ Valor $p$} \\
\hline & & $\mathbf{n}$ & $\%$ & $\mathbf{n}$ & $\%$ & \\
\hline Sexo & $\begin{array}{l}\text { Femenino } \\
\text { Masculino }\end{array}$ & $\begin{array}{l}32 \\
20\end{array}$ & 61,5 & $\begin{array}{l}32 \\
20\end{array}$ & 61,5 & $\begin{array}{l}0,32 \\
0,32\end{array}$ \\
\hline Edad (años) & Mediana & 61 & & 61 & & 0,6 \\
\hline $\begin{array}{l}\text { Variables clínicas } \\
\text { Diagnóstico de implantación del } \\
\text { marcapasos }\end{array}$ & $\begin{array}{l}\text { Bloqueo AV } \\
\text { FA bloqueada } \\
\text { Enfermedad del nódulo } \\
\text { Hipersensibilidad carotídea } \\
\text { Muerte súbita }\end{array}$ & $\begin{array}{r}37 \\
4 \\
4 \\
1 \\
6\end{array}$ & $\begin{array}{r}71,1 \\
7,7 \\
7,7 \\
1,9 \\
11,5\end{array}$ & $\begin{array}{r}37 \\
4 \\
4 \\
1 \\
6\end{array}$ & $\begin{array}{r}71,1 \\
7,7 \\
7,7 \\
1,9 \\
11,5\end{array}$ & $\begin{array}{l}0,33 \\
1,0 \\
0,08 \\
0,61 \\
0,32\end{array}$ \\
\hline Síntomas previos a la implantación & $\begin{array}{l}\text { Asintomático } \\
\text { Disnea } \\
\text { Palpitaciones } \\
\text { Pre-síncope } \\
\text { Síncope }\end{array}$ & $\begin{array}{r}5 \\
23 \\
4 \\
21 \\
19\end{array}$ & $\begin{array}{r}9,6 \\
44,2 \\
7,7 \\
40,4 \\
36,5\end{array}$ & $\begin{array}{r}4 \\
15 \\
8 \\
12 \\
19\end{array}$ & $\begin{array}{r}7,7 \\
28,8 \\
15,4 \\
23,1 \\
36,5\end{array}$ & $\begin{array}{l}0,90 \\
0,90 \\
0,60 \\
0,50 \\
0,43\end{array}$ \\
\hline Enfermedades asociadas & $\begin{array}{l}\text { HTA } \\
\text { Diabetes mellitus } \\
\text { Dislipidemias } \\
\text { Tabaquismo } \\
\text { Enfermedad coronaria }\end{array}$ & $\begin{array}{r}22 \\
7 \\
18 \\
13 \\
10\end{array}$ & $\begin{array}{l}42,3 \\
13,5 \\
34,6 \\
25 \\
19,2\end{array}$ & $\begin{array}{r}24 \\
5 \\
26 \\
17 \\
15\end{array}$ & $\begin{array}{c}46,2 \\
9,6 \\
50 \\
32,7 \\
28,8\end{array}$ & $\begin{array}{l}1,0 \\
0,80 \\
0,10 \\
0,32 \\
0,50\end{array}$ \\
\hline
\end{tabular}

Bloqueo AV: bloqueo aurículo-ventricular completo o de segundo grado.

FA bloqueada: fibrilación auricular con bloqueo aurículo-ventricular.

HTA: hipertensión arterial; $p>0,05$ para la comparación entre grupos de cada una de las variables. 


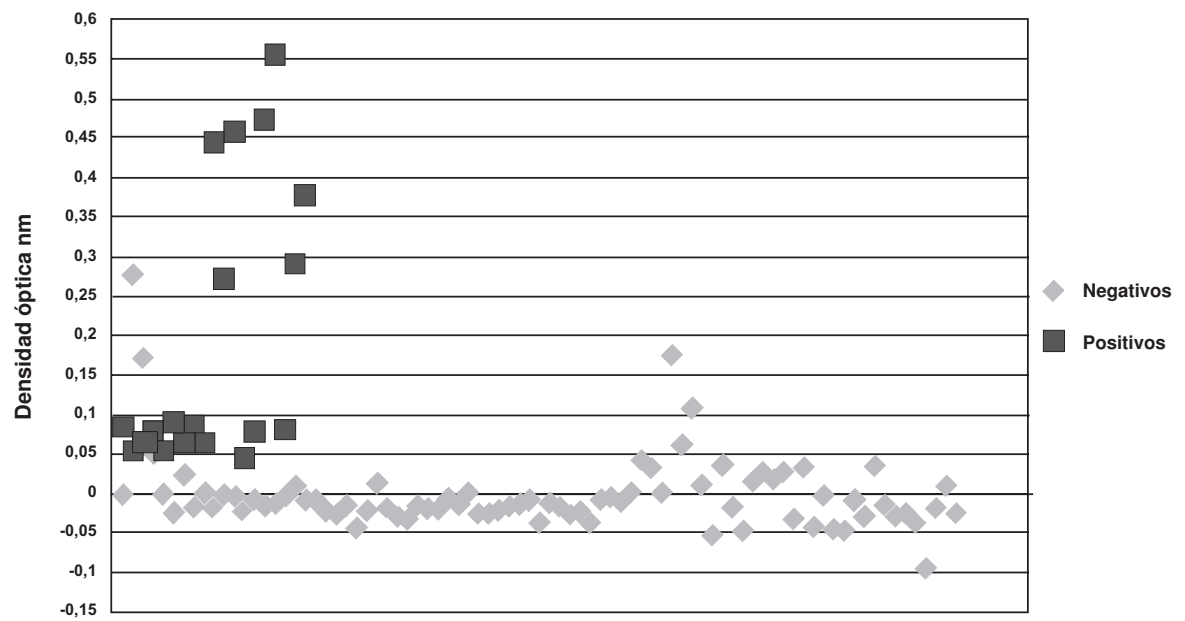

Figura 3. Dispersión de las densidades ópticas de las muestras evaluadas por ELISA para el sde-m2MAChR. Los rombos representan los valores de absorbancia de los sueros considerados como negativos y, los cuadros, los de los considerados positivos. Se establecieron como muestras positivas aquéllas que tuvieran un valor de absorbancia 2,5 veces mayor que el promedio de los controles negativos de cada ensayo.

Cuadro 2. Comparación de la distribución de anticuerpos anti-m2MAChR entre casos y controles.

\begin{tabular}{|c|c|c|c|c|c|c|c|}
\hline & & \multicolumn{2}{|c|}{$\begin{array}{l}\text { Enfermedad de } \\
\text { Chagas }(n=52)\end{array}$} & \multicolumn{2}{|c|}{$\begin{array}{c}\text { Controles } \\
(n=52)\end{array}$} & \multirow[t]{2}{*}{$\mathbf{p}^{1}$} & \multirow[t]{2}{*}{ OR } \\
\hline & & $\%$ & $\mathbf{n}$ & $\%$ & $\mathbf{n}$ & & \\
\hline Anti- tdi-m2MAChR & + & 51,9 & 27 & 19,2 & 10 & 0,0004 & 4,54 \\
\hline Anti- sde-m2MAChR & + & 32,7 & 17 & 3,8 & 2 & 0,0001 & 12,14 \\
\hline $\begin{array}{l}\text { Anti- tdi-m2MAChR y } \\
\text { Anti- sde-m2MAChR }\end{array}$ & + & 15,4 & 8 & 1,9 & 1 & 0,01 & 9,2 \\
\hline
\end{tabular}

Anti-sde-m2MAChR: anticuerpos anti-m2MAChR que reconocen el segundo dominio extracelular del receptor. Anti-tdi-m2MAChR: anticuerpos anti-m2MAChR que reconocen el tercer dominio intracelular del receptor. ${ }^{1} \mathrm{p}$ corregida por Fisher, intervalos de confianza del 95\%

del dispositivo de marcapaso cardiaco. No se evidenciaron diferencias estadísticamente significativas entre la presencia de anticuerpos anti-m2MAChR y el diagnóstico de implantación de marcapasos (no se presentan los datos).

La presencia de anticuerpos contra el m2MAChR no se encontró relacionada en ningún grado con las variables de persona (edad, sexo), lugar (procedencia), anormalidades clínicas previa implantación del marcapaso y funcionamiento del marcapasos.

\section{Discusión}

El presente estudio se basó en la comparación de la frecuencia de presentación de anticuerpos anti-m2MAChR en dos grupos de pacientes cardiópatas con y sin cardiopatía chagásica crónica. El diseño experimental de casos y controles con pareo basado en el diagnóstico de implante del marcapaso, evidenció una mayor probabilidad de presentar anticuerpos anti-m2MAChR en pacientes cardiópatas con infección por $T$. cruzi.

Los resultados obtenidos en las diferentes pruebas realizadas sugieren que las personas con cardiopatía e infección por $T$. cruzi tienen una mayor probabilidad de presentar anticuerpos anti-m2MAChR. El presentar anticuerpos anti-m2MAChR contra el tercer dominio intracelular, es 4,5 veces más probable en casos de cardiopatía chagásica crónica que en pacientes con cardiopatía de otro origen, y es 
12,1 veces más probable presentar anticuerpos anti-m2MAChR que reconocen el segundo dominio extracelular del receptor en casos de cardiopatía chagásica crónica que en pacientes con cardiopatía no asociada a la infección por T. cruzi.

En el análisis de la sintomatología previa a la implantación del marcapasos, los perfiles demográficos y los diagnósticos de implante, no se presentó ninguna diferencia significativa entre quienes presentaban anticuerpos antim2MAChR y aquéllos que no los presentaban (no se presentan los datos). Asimismo, es importante anotar que, a pesar del OR (odds ratio) alto para anticuerpos anti-m2MAChR e infección por T. cruzi, no todos los pacientes cardiópatas seropositivos para T. cruzi, presentaban estos anticuerpos. Por lo tanto, es controversial su relevancia como marcadores de pronóstico de desarrollo de cardiopatía por la infección con T. cruzi.

Los resultados obtenidos en el presente estudio no están en total concordancia con los de otros autores. Altschüller et al. informaron, recientemente, que mediante un modelo de regresión lineal en 67 pacientes con cardiopatía chagásica crónica se evidenció interdependencia entre la presencia de anticuerpos anti-m2MAChR y la disfunción del nódulo sinusal (18). Cabe anotar que en el presente estudio sólo 7,7\% de los pacientes presentaban disfunción sinusal; de éstos, ninguno presentó anticuerpos anti-m2MAChR, mientras que en el estudio mencionado el $46 \%$ de los pacientes presentaba este diagnóstico. Otros autores han sugerido que la presencia de anticuerpos anti-m2MAChR tiene un efecto inhibitorio sobre la función del nervio vago (13). No obstante, en este mismo estudio se concluye que éstos no están asociados con el nivel de disfunción ventricular y, por lo tanto, su relevancia fisiopatológica y pronóstica en el desarrollo de la cardiopatía chagásica continúa sin establecerse. Esta conclusión está acorde con lo planteado recientemente por Talvani, quien sustenta la imposibilidad de asociar la presencia de los anticuerpos anti-m2MAChR con el grado de disfunción cardiaca en ensayos clínicos (20).
En los trabajos realizados hasta la fecha, la frecuencia de anticuerpos contra el segundo dominio extracelular del m2MAChR en pacientes con enfermedad de Chagas, es mucho mayor que la encontrada en el presente estudio: $53 \%$ (en 32 pacientes) (19) y 78\% (en 52 pacientes) (20), en comparación con el 32,7\% (en 52 pacientes) informado aquí. Las frecuencias descritas por otros autores corresponden a pacientes de Argentina y Brasil.

Como algunos autores lo han postulado y como lo podría sugerir el OR (cuadro 2) encontrado en este trabajo, el parásito puede ser el causante de la producción de estos anticuerpos. No obstante, esta idea continúa siendo motivo de discusión.

Diversos autores sostienen la hipótesis de que la respuesta contra el sde-m2MAChR es producto de la reactividad cruzada entre regiones de proteínas ribosómicas del parásito que, por su homología en la secuencia primaria y la presencia de aminoácidos de carga negativa, producen IgG con capacidad de interacción con los receptores m2MAChR y $\beta$-adrenérgicos (23). Esto está en concordancia con la observación de la desaparición del efecto biológico sobre el tejido cardíaco por parte de anticuerpos antim2MAChR, cuando éstos son preincubados con regiones peptídicas de origen parasitario (24). En este mismo sentido, anticuerpos producidos contra el mayor antígeno de superficie del $T$. cruzi- cruzipain reaccionan contra la secuencia peptídica del sde-m2MAChR y compiten por el sitio de unión en el receptor con su antagonista quinuclidinil benzilato (25). Cremanschi informó, en el 2004, la producción de un anticuerpo monoclonal contra $T$. cruzi que reconocía tanto receptores $\beta$-adrenérgicos como m2MAChR en el tejido cardiaco de ratón (23).

En el presente estudio, a pesar de la mayor frecuencia de anticuerpos en casos de cardiopatía chagásica crónica, la hipótesis de que la génesis de estos anticuerpos es producto de la reactividad cruzada entre $T$. cruzi y el receptor no estaría en concordancia con el hallazgo de que $3,8 \%$ de los pacientes del grupo control presentaron anticuerpos anti-m2MAChR. Teniendo en cuenta que estos pacientes nunca 
han entrado en contacto con el parásito (21), existe la posibilidad de que los anticuerpos sean generados por razones ajenas a la infección.

En estudios independientes se ha informado que $40 \%$ de los pacientes con cardiomiopatía dilatada idiopática (19) y 7,5\% de pacientes con fibrilación aurícular idiopática (14) presentan anticuerpos contra el sde-m2MChR. Esto es consistente con lo revisado por $\mathrm{Fu}$ (26), quien describió, a finales de los ochenta, la presencia de anticuerpos contra el sde-m2MChR en pacientes con cardiomiopatía dilatada. Esta variable no fue evaluada en el presente estudio; por lo tanto, no se puede descartar que el común denominador entre los pacientes que presentaron anticuerpos anti-m2MAChR contra el segundo dominio, sea la cardiopatía dilatada.

En el presente estudio se determinó la frecuencia de los anticuerpos contra el tercer dominio intracelular del m2MAChR, por Western blot, utilizando como antígeno una proteína de fusión de $50 \mathrm{kDa}$ que contiene la parte central del tercer dominio intracelular del m2MAChR (25). Los resultados obtenidos por este método mostraron una frecuencia de anticuerpos contra tdi-m2MAChR en el grupo de los casos de $51,9 \%$ y, en el de los controles, de $19,2 \%$, con una diferencia estadísticamente significativa. El tercer dominio intracelular del receptor es importante porque confiere especificidad para el acople de la proteína $\mathrm{G}$ y, además, porque es un sitio blanco de la fosforilación por cinasas (23). Retondaro, en 1996 (27), con un diseño similar y menor número de pacientes, describió una frecuencia de $48 \%$ de reactividad entre pacientes con cardiopatía chagásica crónica y asociación débil entre la presencia anticuerpos anti-m2MAChR y el grado de compromiso cardíaco. La respuesta contra las secuencias peptídicas del tercer dominio intracelular del receptor también ha sido explicada por algunos autores por reactividad cruzada, gracias a la carga negativa de los aminoácidos (27).

No obstante, el hecho de que en el grupo control la frecuencia de los anticuerpos contra el tercer dominio intracelular sea seis veces mayor que la frecuencia de anticuerpos contra el segundo dominio extracelular (cuadro2), permitiría suponer que esta respuesta corresponde a una respuesta inespecífica de destrucción de cardiomiocitos por causas diversas, y el hecho de que sea 4,5 veces más probable encontrar estos anticuerpos en infección por $T$. cruzi estaría explicado por el alto grado de destrucción de tejido que conlleva la infección por este parásito (28).

\section{Agradecimientos}

A Neil Nathanson de la University of Washington, por la donación de anticuerpo monoclonal contra m2MAChR; a Mauricio Rojas de Emory University, por la donación del péptido sintético; a Alberto Moren del Emory Vaccine Center, por el suministro de reactivos; a Irene Cerezo, por el apoyo logístico, y a Gustavo Rey, por el diseño y la digitalización de la base de datos.

\section{Conflicto de intereses}

Los autores de este artículo garantizamos que no tenemos conflictos de intereses.

\section{Financiación}

Este trabajo fue financiado por la División de Investigaciones, sede Bogotá, de la Universidad Nacional de Colombia, DIB Proyecto No20101006346. Programa de Jóvenes Investigadores 2007-Colciencias.

\section{Referencias}

1. Abrams P, Andersson KE, Buccafusco JJ, Chapple C, de Groat WC, Fryer AD, et al. Muscarinic receptors: their distribution and function in body systems, and the implications for treating overactive bladder. J Pharmacol. 2006;148:565-78.

2. Myslivecek J, Nováková M, Palkovits M, Krizanová $\mathbf{O}$, Kvetnanský R. Distribution of mRNA and binding sites of adrenoceptors and muscarinic receptors in the rat heart. Life Sci. 2006;79:112-20.

3. Wang $\mathbf{H}$, Han $\mathbf{H}$, Zhang L, Shi H, Schram G, Nattel $\mathrm{S}$, et al. Expression of multiple subtypes of muscarinic receptors and cellular distribution in the human heart. Mol Pharmacol. 2001;59:1029-36.

4. Pierce KL, Lefkowitz RJ. Classical and new roles of beta-arrestins in the regulation of G-protein-coupled receptors. Nat Rev Neurosci. 2001;2:727-33.

5. Goin JC, Borda ES, Auger S, Storino R, Sterin L. Cardiac M2 muscarinic cholinoceptor activation by human autoantibodies: association with bradycardia. Heart. 1999;82:273-8. 
6. Joensen L, Borda E, Kohout T, Perry S, García G, Sterin L. Trypanosoma cruzi antigen that interacts with the $\beta_{1}-$ adrenergic receptor and modifies myocardial contractile activity. Mol Biochem Parasitol. 2003;127:169-77.

7. Peter JC, Wallukat G, Tugler J, Maurice D, Roegel JC, Briand JP, et al. Modulation of the M2 muscarinic acetylcholine receptor activity with monoclonal anti-M2 receptor antibody fragments. J Biol Chem. 2004;279:55697-706.

8. Borda LE, Gorelik G, Genaro A, Goin JC, Borda ES. Human chagasic IgG interacting with lymphocyte neurotransmitter receptor triggers intracellular signal transduction. FASED J. 1990;4:1661-7.

9. Goin JC, Perez C, Borda E, Sterin BL. Interaction of human chagasic $\lg G$ with the second extracellular loop of the human heart muscarinic acetylholine receptor: functional and pathological implications. FASED J. 1997;10:77-83.

10. Goin JC, Borda E, Leiros CP, Storino R, Sterin-Borda L. Identification of antibodies with muscarinic cholinergic activity in human Chagas' disease: Pathological implications. J Auton Nerv Syst. 1994;47:45-52.

11. Leiros C, Sterin-Borda L, Borda E, Goin JC, Hosey M. Desensitization and sequestration of human M2 $\mathrm{mAChRs}$ by autoantibodies from patients with Chagas'disease. J Biol Chem. 1997;272:12989-93.

12. Retondaro FC, dos Santos Costa PC, Pedrosa RC, Kurtenbach E. Presence of antibodies against the third intracellular loop of the $\mathrm{m} 2$ muscarinic receptor in the sera of chronic chagasic patients. FASEB J. 1999:13;2015-20.

13. Ribeiro AL, Giménez LE, Hernández CC, de Carvalho AC, Teixeira MM, Guedes VC, et al. Early occurrence of anti-muscarinic autoantibodies and abnormal vagal modulation in Chagas disease. Inter J Cardiol. 2007; 117:59-63.

14. Baba A, Yoshikawa T, Fukuda Y, Sugiyama T, Shimada M, Akaishi M, et al. Autoantibodies against M2-muscarinic acetylcholine receptors: new upstream targets in atrial fibrillation in patients with dilated cardiomyopathy. Eur Heart J. 2004;25:1108-15.

15. Del Corsso C, Carvalho AC, Martino HF, Varanda WA. Sera from patients with idiopathic dilated cardiomyopathy decrease $\mathrm{ICa}$ in cardiomyocytes isolated from rabbits. Am J Physiol Heart Circ Physiol. 2004;287:H1928-36.

16. Hernández CC, Barcellos LC, Giménez LE, Cabarcas RA, García S, Pedrosa RC, et al. Human chagasic lgGs bind to cardiac muscarinic receptors and impair L-type Ca2+ currents. Cardiovasc Res. 2003;58:55-65.

17. Ribeiro AL, Giménez LE, Hernández CC, de Carvalho AC, Teixeira MM, Guedes VC, et al. Early occurrence of anti-muscarinic autoantibodies and abnormal vagal modulation in Chagas disease. Int $\mathrm{J}$ Cardiol. 2007; 117:59-63.
18. Corrêa MB, Pedrosa RC, Pereira BB, Corrêa WB, Medeiros A, Costa PC. Chronic Chagas disease patients with sinus node dysfunction: is the presence of $\operatorname{lgG}$ antibodies with muscarinic agonist action independent of left ventricular dysfunction. Rev Soc Bras Med Trop. 2007;40:665-71.

19. Medei E, Pedrosa RC, Benchimol PR, Costa PC, Hernández CC, Chaves EA, et al. Human antibodies with muscarinic activity modulate ventricular repolarization: basis for electrical disturbance. Int $\mathrm{J}$ Cardiol. 2007;115:373-80.

20. Talvani A, Rocha MO, Ribeiro AL, Borda E, SterinBorda L, Teixeira MM. Levels of anti-M2 and anti-beta1 autoantibodies do not correlate with the degree of heart dysfunction in Chagas' heart disease. Microbes Infect. 2006;8:2459-64.

21. Mora G, Echeverry M, Rey G, López M, Posada L, Rivas F. Frecuencia de anticuerpos anti-Trypanosoma cruzi en pacientes portadores de marcapasos de la Clínica San Pedro Claver de Bogotá. Biomédica. 2007;27:483-9.

22. Luetje C, Brumwel C, Gainer M, Peterson G, Schimerlik $\mathbf{M}$, Nathanson $\mathbf{N}$. Isolation and characterization of monoclonal antibodies specific for the cardiac muscarinic acetylcholine receptor. Biochemestry. 1987;26:6892-6.

23. Cremaschi G, Fernández MM, Gorelik G, Goin JC, Fossati CA, Zwirner NW, et al. Modulatory effects on myocardial physiology induced by an anti-Trypanosoma cruzi monoclonal antibody involve recognition of major antigenic epitopes from beta1-adrenergic and M2muscarinic cholinergic receptors without requiring receptor cross-linking. J Neuroimmunol. 2004;153:99107.

24. Masuda MO, Levin M, De Oliveira SF, Dos Santos PC, Bergami PL, Dos Santos NA, et al. Functionally active cardiac antibodies in chronic Chagas' disease are specifically blocked by Trypanosoma cruzi antigens. FASEB J. 1998;12:1551-8.

25. Sterin-Borda L, Giordanengo L, Joensen L, Gea S. Cruzipain induces autoantibodies against cardiac muscarinic acetylcholine receptors. Functional and pathological implications. Eur J Immunol. 2003;33: 2459-68.

26. Fu ML. Characterization of anti-heart M2 muscarinic receptor antibodies -a combined clinical and experimental study. Mol Cell Biochem. 1996;163:343-7.

27. Retondaro FC, Dos Santos PC, Pedrosa RC, Kurtenbach E. Presence of antibodies against the third intracellular loop of the $\mathrm{m} 2$ muscarinic receptor in the sera of chronic chagasic patients. FASEB J. 1999;13:2015-20.

28. Marin-Neto JA, Cunha-Neto E, Maciel BC, Simões MV. Pathogenesis of chronic Chagas heart disease. Circulation. 2007;115:1109-23. 